\section{Validade de constructo de escala de apoio social do Medical Outcomes Study adaptada para o português no Estudo Pró-Saúde}

\author{
Construct validity of the Medical Outcomes \\ Study's social support scale adapted \\ to Portuguese in the Pró-Saúde Study
}

\author{
Rosane Harter Griep 1 \\ Dóra Chor 2 \\ Eduardo Faerstein 3 \\ Guilherme L. Werneck 3,4 \\ Cláudia S. Lopes ${ }^{3}$
}

\footnotetext{
${ }^{1}$ Escola de Enfermagem Anna Nery, Universidade Federal do Rio de Janeiro, Rio de Janeiro, Brasil.

2 Escola Nacional de Saúde Pública, Fundação Oswaldo Cruz, Rio de Janeiro, Brasil.

3 Instituto de Medicina Social, Universidade do Estado do Rio de Janeiro, Rio de Janeiro, Brasil. 4 Faculdade de Medicina, Universidade Federal do Rio de Janeiro, Rio de Janeiro, Brasil.

Correspondência R. H. Griep Departamento de Enfermagem em Saúde Pública, Escola de Enfermagem Anna Nery, Universidade Federal do Rio de Janeiro. Rua Afonso Cavalcante 275 , Rio de Janeiro, $R J$ 20211-110, Brasil. rohgriep@terra.com.br
}

\begin{abstract}
This paper evaluates the construct validity of the Medical Outcomes Study's social support scale adapted to Portuguese, when utilized in a cohort study among non-faculty civil servants at a university in Rio de Janeiro, Brazil (PróSaúde Study). Baseline data were obtained in 1999, when 4,030 participants (92.0\% of those eligible) completed a multidimensional self-administered questionnaire at the workplace. From the original scale's five social support dimensions, factor analysis of the data extracted only three dimensions: positive social interaction/affective support; emotional/information support; and material support. We estimated associations between social support dimensions and socio-demographic, health, and well beingrelated characteristics. We confirmed the hypotheses that less isolated individuals, those with better self-rated health, those who reported more participation in group activities, and those with no evidence of common mental disorders reported better perception of social support. In conclusion, we found good evidence for a high construct validity of this scale, supporting its use in future analyses in the Pró-Saúde Study and in similar population groups.
\end{abstract}

Social Support; Cohort Studies; Questionnaires

\section{Introdução}

Cassel ${ }^{1}$ e Cobb ${ }^{2}$ sugeriram que a ruptura de laços sociais afeta os sistemas de defesa do organismo de tal maneira que o indivíduo se torna mais suscetível a doenças. De acordo com essa idéia, os laços sociais teriam influência na manutenção da saúde, favorecendo condutas adaptativas em situações de estresse. Vários estudos investigaram a influência desses laços no risco de adoecer e morrer, e seus resultados indicam que dispor de uma rede social e receber ajuda dos indivíduos que pertencem a essa rede beneficiam a saúde e o bem-estar 3,4,5,6,7,8,9, $10,11,12$. Ao mesmo tempo, outros estudos investigaram a confiabilidade e a validade de diferentes instrumentos de medida de rede e apoio social 13,14,15,16.

Os primeiros instrumentos de medida de vínculos sociais consistiam de índices simples, freqüentemente abrangendo apenas a presença ou ausência de cônjuge, a disponibilidade de um confidente durante situações de crise, a composição da família e/ou a participação em atividades sociais 16 . Essas medidas isoladas, apesar de terem se mostrado boas preditoras das condições de saúde e também do risco de morrer, não informam sobre a natureza desses relacionamentos e não detalham os mecanismos através dos quais os componentes da rede social funcionam como sistema de apoio 14,16. $\mathrm{O}$ desenvolvimento de indicadores mais abran- 
gentes, multidimensionais, que levam em conta aspectos funcionais das relações sociais, envolveu constructos mais amplos, com maior nível de abstração e, conseqüentemente, grande diversidade conceitual 16 .

Dentre os constructos envolvendo laços sociais, destacam-se os conceitos de rede e apoio social.

Rede social (social network) pode ser definida como o grupo de pessoas com as quais o indivíduo mantém contato ou alguma forma de vínculo social 16, que podem ou não oferecer ajuda em diversas situações ao longo da vida.

Apoio social (social support) diz respeito aos recursos postos à disposição por outras pessoas em situações de necessidade 17 e pode ser medido através da percepção individual do grau com que relações interpessoais correspondem a determinadas funções (por exemplo, apoio emocional, material e afetivo) 18 .

Vários questionários foram propostos com o objetivo de medir rede e apoio social, refletindo uma diversidade de conceitos e definições subjacentes 14,19, o que dificulta a comparação dos resultados das investigações conduzidas em diferentes populações. Como se tem grande interesse nessa comparação, é desejável a utilização de instrumentos de medida adaptados para utilização em contextos diversos daqueles para os quais foram inicialmente concebidos. Torna-se igualmente importante a avaliação das propriedades de mensuração desses instrumentos de medida adaptados aos novos contextos 20 .

A inexistência de instrumentos brasileiros validados para a medida de apoio social motivou a escolha da escala de apoio social utilizada no Medical Outcomes Study (MOS) 18, para utilização em um estudo de coorte de funcionários de uma universidade no Rio de Janeiro, Brasil (Estudo Pró-Saúde). Em publicações anteriores 21,22,23,24, foram apresentadas várias etapas da avaliação do processo de tradução desse instrumento do inglês para o português, e sua adaptação ao contexto em que foi aplicado. Neste artigo, apresentamos aspectos da validade de constructo da escala aplicada no Estudo Pró-Saúde. A validade é estabelecida a partir de um conjunto de técnicas que buscam avaliar o grau com que uma medida empírica reflete adequadamente o significado real do conceito considerado 25 . Especificamente, a validade de constructo refere-se às relações entre os conceitos supostamente captados pelo instrumento que se deseja avaliar e outros conceitos, atributos e características relacionados ao constructo em estudo, de acordo com hipóteses previamente estabelecidas 25 .

\section{Métodos}

\section{População de estudo}

Foram estudados os participantes da fase 1 (agosto a novembro de 1999) de um estudo longitudinal - o Estudo Pró-Saúde, cujo principal objetivo é estudar a associação entre determinantes sociais e diversos desfechos de saúde 26 . Nessa etapa, de um universo de 4.448 funcionários técnico-administrativos efetivos de uma universidade no Rio de Janeiro, 4.030 (91,0\% da população-alvo) aderiram ao estudo. Foram excluídos os aposentados, os cedidos a outras instituições e os licenciados sem vencimentos. Os funcionários completaram, no ambiente de trabalho, um questionário autopreenchível e tiveram peso e estatura aferidos.

\section{A escala de apoio social}

A escala de apoio social escolhida foi elaborada, originalmente, para o MOS 18. Trata-se de um estudo que abrangeu 2.987 adultos, usuários de serviços de saúde em Boston, Chicago e Los Angeles, nos Estados Unidos, que apresentavam uma ou mais das seguintes doenças crônicas: hipertensão, diabetes, doença coronariana ou depressão. Na sua forma original, esse instrumento foi concebido para abranger cinco dimensões de apoio social (Tabela 1): material (quatro perguntas) - provisão de recursos práticos e ajuda material; afetiva (três perguntas) - demonstrações físicas de amor e afeto; interação social positiva (quatro perguntas) contar com pessoas com quem relaxar e divertir-se; emocional (quatro perguntas) - habilidade da rede social em satisfazer as necessidades individuais em relação a problemas emocionais, por exemplo situações que exijam sigilo e encorajamento em momentos difíceis da vida; informação (quatro perguntas) - contar com pessoas que aconselhem, informem e orientem. Para todas as perguntas, cinco opções de resposta foram apresentadas: 1 ("nunca"); 2 ("raramente”); 3 (“às vezes"); 4 (“quase sempre”) e 5 ("sempre").

\section{Procedimentos de análise dos dados}

\section{- Validade de constructo}

\section{a) Análise fatorial}

A capacidade do instrumento em discriminar, em nossa população de estudo, as dimensões subjacentes à versão original da escala foi avaliada por meio da análise fatorial, aplicando-se 
Questionário de apoio social, itens e dimensões propostas na versão original aplicada no Medical Outcomes Study، adaptadas para o português.

\begin{tabular}{|c|c|}
\hline Tipo de apoio & $\begin{array}{l}\text { Item } \\
\text { Se você precisar, com que freqüência conta com alguém... }\end{array}$ \\
\hline \multirow[t]{4}{*}{ Material } & que o ajude, se ficar de cama? \\
\hline & para levá-lo ao médico? \\
\hline & para ajudá-lo nas tarefas diárias, se ficar doente? \\
\hline & para preparar suas refeições, se você não puder prepará-las? \\
\hline \multirow[t]{3}{*}{ Afetivo } & que demonstre amor e afeto por você? \\
\hline & que the dê um abraço? \\
\hline & que você ame e que faça você se sentir querido? \\
\hline \multirow[t]{4}{*}{ Emocional* } & para ouvi-lo, quando você precisar falar? \\
\hline & em quem confiar ou para falar de você ou sobre seus problemas? \\
\hline & para compartilhar suas preocupações e medos mais íntimos? \\
\hline & que compreenda seus problemas? \\
\hline \multirow[t]{4}{*}{ Informação* } & para dar bons conselhos em situações de crise? \\
\hline & para dar informação que o ajude a compreender uma determinada situação? \\
\hline & de quem você realmente quer conselhos? \\
\hline & para dar sugestões de como lidar com um problema pessoal? \\
\hline \multirow[t]{4}{*}{ Interação social positiva } & com quem fazer coisas agradáveis? \\
\hline & com quem distrair a cabeça? \\
\hline & com quem relaxar? \\
\hline & para se divertir junto? \\
\hline
\end{tabular}

* Dimensões não discriminadas em avaliações empíricas do estudo original, tratadas como uma única dimensão 18.

o método dos eixos principais (Principal Axis Factoring) para a extração de fatores 27 . Tratase de uma técnica estatística multivariada que analisa inter-relações entre variáveis, de forma que estas possam ser representadas por um conjunto de fatores ou dimensões que agregam, tanto quanto possível, as variáveis iniciais 27 . Para determinar o número de fatores que deveriam ser extraídos, dois critérios foram utilizados: o número de fatores com autovalor (eigenvalue) superior a um e o Cattell's Scree test 27. Em uma segunda fase, realizou-se rotação ortogonal varimax para simplificar a interpretação dos fatores e minimizar o número de itens com grande peso em apenas um fator. Como exploração inicial da adequação dos itens ao procedimento de análise fatorial, foram utilizados o índice de Kaiser Meyer-Olkin (KMO) e o teste de esfericidade de Bartlett (BTS), os quais testam a hipótese nula de matriz identidade, ou seja, de que além da correlação perfeita (diagonal principal) não há correlação entre as variáveis 27 .

\section{b) Correlação item-escala}

Foram construídos escores para cada uma das três dimensões de apoio social sugeridas pela análise fatorial. Esses escores foram padronizados segundo o número de perguntas que fazem parte de cada dimensão. Assim, a soma de pontos obtidos pelas perguntas de cada uma das dimensões foi dividida pelo escore máximo possível na mesma dimensão (que variava de acordo com o número de perguntas). O resultado da razão (total de pontos obtidos/pontuação máxima da dimensão) foi multiplicado por 100. Os funcionários que deixaram de responder a qualquer pergunta de determinada dimensão foram excluídos do cálculo do escore desta 22 .

Com o objetivo de avaliar o grau de correlação entre cada item e sua respectiva dimensão, utilizou-se o coeficiente de correlação de Pearson. Além disso, correlações item-escala corrigidas foram obtidas: a pontuação dos itens que fazem parte de cada dimensão foi somada, excluindo-se o item analisado, a fim de evitar o 
aumento artificial do valor do coeficiente. Dois critérios foram adotados na avaliação dessas correlações: correlação $\geq 0,40$ de todos os itens com suas respectivas dimensões 28; estimativa do coeficiente de correlação do item com o escore de sua dimensão ter valor de, pelo menos, dois erros-padrão $(1 / \sqrt{n})$ maior do que o mesmo coeficiente entre aquele item e qualquer outra dimensão 18,28 .

\section{c) Hipóteses avaliadas}

A investigação empírica de diferentes hipóteses que relacionam o escore de apoio social a outras características descritas na literatura epidemiológica foi também realizada 18,29,30. Para o teste dessas hipóteses, explorou-se a associação entre apoio social "alto" ou "baixo" (ponto de corte estabelecido pela mediana da distribuição de cada dimensão) e diversas características apresentadas na Tabela 2. As associações foram estimadas mediante razões de chances (odds ratio - OR), com intervalos de 95\% de confiança.

\section{- Consistência interna das dimensões da escala}

Os valores relativos à consistência interna da escala global e das três dimensões de apoio social foram estimados por meio do coeficiente alpha de Cronbach 25. Esses coeficientes foram também estimados excluindo-se cada item de sua dimensão, de tal forma que, se ocorresse um aumento superior a 0,10 no valor do coeficiente alpha de Cronbach após a exclusão do item específico, a consistência interna daquela dimensão estaria sendo prejudicada, indicando-se a avaliação de sua exclusão da escala.

Todas as análises foram realizadas utilizando-se o programa estatístico SPSS versão 10.0.

\section{Aspectos éticos}

Garantiu-se aos participantes a participação voluntária no estudo. Os funcionários preenchiam o questionário somente após a leitura e assinatura do Termo de Consentimento, documento no qual estava registrado o compromis-

Tabela 2

Hipóteses de validade de constructo e características avaliadas.

\begin{tabular}{|c|c|c|}
\hline Características & Hipóteses avaliadas & Características investigadas \\
\hline Sócio-demográficas & $\begin{array}{l}\text { Entre as mulheres, entre os mais jovens, } \\
\text { entre os indivíduos de escolaridade e renda } \\
\text { mais altas são mais freqüentes altos escores } \\
\text { de apoio social. }\end{array}$ & $\begin{array}{l}\text { Gênero, faixa etária, escolaridade e renda per capita } \\
\text { em salários mínimos. }\end{array}$ \\
\hline \multirow[t]{3}{*}{ De rede social } & $\begin{array}{l}\text { Indivíduos com maior número de parentes e } \\
\text { amigos íntimos referidos têm maior chance } \\
\text { de apresentar altos escores de apoio social. }\end{array}$ & $\begin{array}{l}\text { Número de parentes e amigos íntimos (definidos como } \\
\text { aqueles com quem se sentem à vontade e podem } \\
\text { falar sobre quase tudo). }\end{array}$ \\
\hline & $\begin{array}{l}\text { Indivíduos que participam de atividades } \\
\text { sociais em grupo têm mais chance de } \\
\text { apresentar altos escores de apoio social. }\end{array}$ & $\begin{array}{l}\text { Participação nos últimos } 12 \text { meses em atividades } \\
\text { esportivas ou artísticas em grupo, trabalho voluntário } \\
\text { ou em atividades religiosas. }\end{array}$ \\
\hline & $\begin{array}{l}\text { Indivíduos casados ou que não moram } \\
\text { sozinhos têm chances mais elevadas de } \\
\text { apresentar altos escores de apoio social. }\end{array}$ & $\begin{array}{l}\text { Situação conjugal (casado/não casado); condição } \\
\text { de morar sozinho. }\end{array}$ \\
\hline \multirow[t]{2}{*}{ De condições de saúde física } & $\begin{array}{l}\text { Indivíduos com melhor percepção sobre seu } \\
\text { estado de saúde auto-referido apresentam } \\
\text { maiores escores de apoio social. }\end{array}$ & $\begin{array}{l}\text { Pergunta: De um modo geral, em comparação a } \\
\text { pessoas da sua idade, como você considera seu } \\
\text { próprio estado de saúde? }\end{array}$ \\
\hline & $\begin{array}{l}\text { Indivíduos com menor número de doenças } \\
\text { crônicas referidas têm maior chance de } \\
\text { apresentar escores altos de apoio social. }\end{array}$ & Número referido de doenças crônicas. \\
\hline De saúde mental & $\begin{array}{l}\text { Indivíduos classificados como negativos para } \\
\text { transtornos mentais comuns têm maior chance } \\
\text { de apresentar altos escores de apoio social. }\end{array}$ & $\begin{array}{l}\text { Escala de avaliação de transtornos mentais comuns - } \\
\text { GHQ-12 (foram considerados "positivos" aqueles que } \\
\text { responderam afirmativamente a pelo menos três dos } \\
12 \text { itens da escala) } 36 \text {. Esse questionário autopreenchível } \\
\text { foi validado na sua versão original } 37 \text { e na sua versão } \\
\text { brasileira } 38 \text {. }\end{array}$ \\
\hline
\end{tabular}


so do grupo de pesquisa envolvido no Estudo Pró-Saúde de não utilizar os resultados individuais para qualquer finalidade administrativa. Além disso, a identificação do questionário era feita por intermédio de senha numérica, e as respostas foram analisadas apenas como estatísticas agrupadas. Por outro lado, os aferidores e digitadores também assinaram Termo de Sigilo, em que se comprometiam a não divulgar quaisquer informações contidas nos questionários. O estudo foi aprovado pelo Comitê de Ética da universidade onde a investigação foi desenvolvida.

\section{Resultados}

\section{Características da população de estudo}

A população de estudo tinha em média 40 anos (DP $=8,81$ ); 56,0\% eram mulheres; $61,0 \%$ eram casados; $24,0 \%$ tinham cursado o ensino fundamental, $36,0 \%$ cursaram o ensino médio e $40,0 \%$ tinham completado curso superior. A média da renda domiciliar per capita dos funcionários era 5,7 salários mínimos ( $\mathrm{DP}=4,5$ salários mínimos), conforme valor vigente na época do estudo (cada salário mínimo correspondia a $\mathrm{R} \$ 136,00)$.

\section{Validade de constructo}

\section{a) Matriz de correlação e análise fatorial da escala de apoio social}

As correlações entre os itens apresentaram variação entre 0,30 e 0,80 . O teste de esfericidade de Bartlett rejeitou a hipótese nula de que a matriz de correlação dos dados fosse uma matriz identidade $(\mathrm{p}<0,001)$ e o teste KMO foi igual a 0,967. Esses resultados indicam que houve excelente adequação da matriz dos dados à análise fatorial.

De acordo com os procedimentos da análise fatorial, apenas três fatores (dimensões) foram mantidos. Os autovalores dos três fatores foram iguais a 15,47, 1,95 e 1,48, respectivamente, e a proporção da variância explicada por cada um foi estimada em $59,1 \%, 7,4 \%$ e $5,7 \%$, respectivamente. Portanto, esses três fatores explicaram, em conjunto, $72,0 \%$ da variância encontrada nos dados (Tabela 3 ).

Após a rotação varimax das cargas dos fatores, nove itens tiveram cargas mais altas no fator I; seis itens, no fator II; e quatro itens, no fator III. O primeiro fator abrangeu todos os itens de apoio afetivo e de interação social positiva das dimensões originais do questionário. Além disso, dois itens originalmente pertencentes à escala de apoio emocional ("alguém para compartilhar preocupações e medos mais íntimos” e "alguém que compreenda seus problemas") apresentaram cargas mais altas nesse fator. Esses itens também apresentaram altas cargas no fator II, que incluiu os itens remanescentes do apoio emocional e também todos os itens de apoio de informação das dimensões originais. O terceiro fator, de maneira semelhante ao que ocorre na versão original, incluiu todos os itens de apoio material (Tabela 3 ).

\section{b) Correlação entre os itens e as dimensões de apoio social}

Escores globais de cada dimensão de apoio social foram calculados, com suas respectivas médias e desvios-padrão. Foram estimados os coeficientes de correlação de Pearson entre cada item e sua respectiva dimensão (Tabela 4). A correlação item-escala corrigida variou entre 0,71 e 0,81 na dimensão de apoio afetivo/interação social positiva; entre 0,76 a 0,84 na dimensão de apoio informação/emocional; e entre 0,60 e 0,71 na dimensão de apoio material. O item "para compartilhar preocupações e medos mais íntimos” apresentou a correlação item-escala corrigida mais alta com a dimensão emocional/informação, enquanto o item "que compreenda seus problemas" apresentou correlações semelhantes nas dimensões afetivo/interação social positiva e emocional/informação.

\section{c) Hipóteses avaliadas}

Os resultados empíricos das hipóteses testadas encontram-se na Tabela 5. Observou-se que os funcionários mais jovens, os casados, aqueles que não moram sós, os que praticam atividades físicas ou artísticas ou trabalho voluntário, aqueles com melhor estado de saúde auto-referido e os que não foram classificados como positivos para transtornos mentais comuns tiveram chances significativamente maiores de perceberem apoio social mais elevado. Os homens, comparados às mulheres, apresentaram maior chance de perceber alto apoio afetivo/interação social positiva e apoio material. Observou-se, ainda, a presença de gradiente nas medidas de associação entre escolaridade, renda familiar em salários mínimos per capita, número de parentes e amigos íntimos e número de doenças crônicas e as três dimensões de apoio social, com teste de tendência linear estatisticamente significativo para todas essas variáveis $(p<0,01)$. Por exemplo, no caso da associa- 
Carga dos fatores relativos aos itens componentes de apoio social. Estudo Pró-Saúde, 1999.

\begin{tabular}{|c|c|c|c|}
\hline Itens & $\begin{array}{c}\text { Fator } 1 \\
\text { Afetivo + interação } \\
\text { social positival }\end{array}$ & $\begin{array}{c}\text { Fator } 2 \\
\text { Emocional + } \\
\text { informação }\end{array}$ & $\begin{array}{l}\text { Fator } 3 \\
\text { Materia }\end{array}$ \\
\hline \multicolumn{4}{|l|}{ Se você precisar, com que freqüência conta com alguém... } \\
\hline com quem fazer coisas agradáveis? & 0,826 & 0,306 & 0,204 \\
\hline com quem distrair a cabeça? & 0,799 & 0,388 & 0,261 \\
\hline com quem relaxar? & 0,844 & 0,400 & 0,300 \\
\hline para se divertir junto? & 0,703 & 0,309 & 0,308 \\
\hline que você ame e que faça você se sentir querido? & 0,722 & 0,217 & 0,251 \\
\hline que the dê um abraço? & 0,641 & 0,405 & 0,310 \\
\hline que demonstre amor e afeto por você? & 0,492 & 0,345 & 0,394 \\
\hline para compartilhar suas preocupações e medos mais íntimos? & 0,822 & 0,723 & 0,323 \\
\hline que compreenda seus problemas? & 0,753 & 0,587 & 0,170 \\
\hline em quem confiar ou para falar de você ou sobre seus problemas? & 0,540 & 0,821 & 0,294 \\
\hline para ouvi-lo, quando você precisar falar? & 0,321 & 0,779 & 0,466 \\
\hline para dar bons conselhos em situações de crise? & 0,305 & 0,904 & 0,447 \\
\hline para dar sugestões de como lidar com um problema pessoal? & 0,680 & 0,777 & 0,253 \\
\hline de quem você realmente quer conselhos? & 0,623 & 0,747 & 0,288 \\
\hline para dar informação que o ajude a compreender uma determinada situação? & 0,531 & 0,598 & 0,238 \\
\hline que o ajude, se ficar de cama? & 0,157 & 0,519 & 0,917 \\
\hline para levá-lo ao médico? & 0,312 & 0,640 & 0,883 \\
\hline para ajudá-lo nas tarefas diárias, se ficar doente? & 0,540 & 0,239 & 0,723 \\
\hline para preparar suas refeições, se você não puder prepará-las? & 0,547 & 0,093 & 0,658 \\
\hline Autovalores antes da rotação & 15,47 & 1,95 & 1,48 \\
\hline Percentual de variância explicada & 59,05 & 7,44 & 5,67 \\
\hline
\end{tabular}

Método de extração dos fatores: eixos principais; método de rotação: varimax com normalização de Kaiser Meyer-Olkin.

ção entre número de parentes e amigos íntimos e apoio afetivo/interação social positiva, a chance de perceber alto apoio foi cerca de três vezes maior (OR = 3,3; IC95\%: 2,2-4,9) entre aqueles que relataram um a dois amigos, e cerca de dez vezes maior (OR $=10,3$; IC95\%: 6,9$15,4)$ entre os funcionários que relataram oito ou mais, quando comparados àqueles que referiram nenhum parente ou amigo. Esse mesmo padrão, embora com associações mais fracas, foi observado para os funcionários com níveis progressivamente maiores de renda per capita e escolaridade, quando comparados aos de níveis mais baixos dessas características.

Em relação às doenças crônicas referidas, a chance de perceber alto apoio social foi cerca de $70,0 \%$ maior entre aqueles que relataram nenhuma doença, e cerca de $40,0 \%$ maior entre os funcionários que relataram de uma a duas doenças, quando comparados àqueles que referiram três ou mais doenças crônicas, para as três dimensões avaliadas.
Participar de atividades religiosas durante os 12 meses anteriores à pesquisa esteve associado às dimensões de apoio afetivo/interação social positiva e apoio emocional/informação. Assim, respectivamente, nas duas dimensões citadas, os funcionários que referiram freqüentar "uma vez por semana ou mais" tiveram chance $20,0 \%$ maior (OR = 1,2; IC95\%: 1,0-1,5) e 30,0\% maior $(O R=1,3$; IC95\%: $1,1-1,6)$ de referirem alto apoio social, e os que relataram ter freqüentado "duas a três vezes por mês ou algumas vezes nos últimos 12 meses” tiveram chance $20,0 \%$ maior (OR = 1,2; IC95\%: 1,0-1,4) de referirem alto apoio social, quando comparados aos funcionários que responderam "ter participado uma vez no ano ou menos".

\section{Consistência interna}

A média dos escores foi de 83,3 para a dimensão de interação social positiva/apoio afetivo; 78,6 para a dimensão emocional/informação e 
Média de cada item, coeficiente de correlação de Pearson entre os itens e respectivas dimensões de apoio social e coeficiente alpha de Cronbach. Estudo Pró-Saúde, 1999.

\begin{tabular}{|c|c|c|c|c|c|c|c|}
\hline Itens & Média & $\begin{array}{l}\text { Desvio- } \\
\text { padrão }\end{array}$ & $\begin{array}{l}\text { Afetivo e } \\
\text { interação } \\
\text { social } \\
\text { positiva }\end{array}$ & $\begin{array}{l}\text { Emocional e } \\
\text { informação }\end{array}$ & Material & $\begin{array}{l}\text { Alpha de } \\
\text { Da dimensão } \\
\text { se item } \\
\text { excluído }\end{array}$ & $\begin{array}{l}\text { Cronbach } \\
\text { Da dimensão }\end{array}$ \\
\hline \multicolumn{8}{|l|}{$\begin{array}{l}\text { Se você precisar, com que freqüência } \\
\text { conta com alguém... }\end{array}$} \\
\hline com quem fazer coisas agradáveis? & 4,06 & 1,08 & $0,80 *$ & 0,69 & 0,55 & 0,91 & \\
\hline com quem distrair a cabeça? & 3,97 & 1,11 & $0,79 *$ & 0,73 & 0,57 & 0,91 & \\
\hline com quem relaxar? & 3,95 & 1,18 & $0,81^{\star}$ & 0,72 & 0,57 & 0,91 & \\
\hline para se divertir junto? & 4,14 & 1,09 & $0,75^{\star}$ & 0,65 & 0,54 & 0,91 & \\
\hline $\begin{array}{l}\text { que você ame e que faça você } \\
\text { se sentir querido? }\end{array}$ & 4,29 & 1,06 & $0,72^{\star}$ & 0,60 & 0,49 & 0,92 & \\
\hline que the dê um abraço? & 4,27 & 1,05 & $0,77^{\star}$ & 0,70 & 0,57 & 0,91 & \\
\hline que demonstre amor e afeto por você? & 4,45 & 0,95 & $0,71^{\star}$ & 0,67 & 0,62 & 0,92 & 0,92 \\
\hline $\begin{array}{l}\text { para compartilhar suas preocupações } \\
\text { e medos mais íntimos? }\end{array}$ & 3,72 & 1,37 & 0,73 & $0,80^{*}$ & 0,61 & 0,93 & \\
\hline que compreenda seus problemas? & 3,88 & 1,14 & 0,76 & $0,78^{\star}$ & 0,55 & 0,93 & \\
\hline $\begin{array}{l}\text { em quem confiar ou para falar de } \\
\text { você ou sobre seus problemas? }\end{array}$ & 4,00 & 1,20 & 0,70 & $0,82^{\star}$ & 0,59 & 0,93 & \\
\hline $\begin{array}{l}\text { para ouvi-lo, quando você } \\
\text { precisar falar? }\end{array}$ & 4,11 & 1,14 & 0,64 & $0,76^{*}$ & 0,65 & 0,93 & \\
\hline $\begin{array}{l}\text { para dar bons conselhos em } \\
\text { situações de crise? }\end{array}$ & 3,98 & 1,24 & 0,60 & $0,77^{*}$ & 0,62 & 0,93 & \\
\hline $\begin{array}{l}\text { para dar sugestões de como lidar } \\
\text { com um problema pessoal? }\end{array}$ & 3,82 & 1,24 & 0,71 & $0,84^{*}$ & 0,60 & 0,93 & \\
\hline $\begin{array}{l}\text { de quem você realmente } \\
\text { quer conselhos? }\end{array}$ & 3,86 & 1,24 & 0,68 & $0,80 *$ & 0,59 & 0,93 & \\
\hline $\begin{array}{l}\text { para dar informação que o ajude } \\
\text { a compreender uma determinada } \\
\text { situação? }\end{array}$ & 4,03 & 1,07 & 0,70 & $0,76^{\star}$ & 0,59 & 0,93 & 0,94 \\
\hline que o ajude, se ficar de cama? & 4,10 & 1,29 & 0,50 & 0,57 & $0,67 *$ & 0,78 & \\
\hline para levá-lo ao médico? & 3,88 & 1,41 & 0,56 & 0,64 & $0,68^{\star}$ & 0,78 & \\
\hline $\begin{array}{l}\text { para ajudá-lo nas tarefas diárias, } \\
\text { se ficar doente? }\end{array}$ & 4,09 & 1,22 & 0,59 & 0,61 & $0,71^{\star}$ & 0,81 & \\
\hline $\begin{array}{l}\text { para preparar suas refeições, se você } \\
\text { não puder prepará-las? }\end{array}$ & 4,07 & 1,18 & 0,53 & 0,48 & $0,60^{\star}$ & 0,77 & 0,83 \\
\hline
\end{tabular}

* Correlação item-escala corrigida (correlação entre o item específico e os outros itens da mesma dimensão); erro padrão = 0,016.

80,8 para a dimensão apoio material. A média da escala global foi 80,8 . A proporção de dados faltantes foi $1,7 \%, 1,9 \%, 2,0 \%$ e $3,2 \%$, respectivamente, para os escores de apoio material, afetivo/interação social positiva, emocional/informação e escala global.

O coeficiente alpha de Cronbach foi igual ou maior do que 0,83 para todas as dimensões. Observou-se, também, que não ocorreram variações na consistência interna das dimensões, caso algum dos itens fosse excluído das mesmas, mantendo-se, assim, o conjunto original de perguntas (Tabela 4 ).

\section{Discussão}

As pontuações médias das dimensões da escala de apoio social em nosso estudo foram consideravelmente mais elevadas do que as médias encontradas por Sherbourne \& Stewart 18 no estudo norte-americano. Essas diferenças são esperadas em virtude dos contextos sócio-econômico e cultural nas duas populações. Ademais, no estudo norte-americano, participaram indivíduos mais idosos (média de 55 anos de idade), 61,0\% eram mulheres, e todos eram usuários de serviços de saúde e portadores de doenças crônicas.

Em investigação anterior, foi estimado um bom nível de confiabilidade desse instrumento 
Associação entre variáveis selecionadas* e alto apoio social em suas três dimensões. Estudo Pró-Saúde, 1999.

\begin{tabular}{|c|c|c|c|c|c|c|}
\hline \multirow[t]{2}{*}{ Variáveis } & \multicolumn{2}{|c|}{$\begin{array}{l}\text { Apoio afetivo/ } \\
\text { interação social positiva }\end{array}$} & \multicolumn{2}{|c|}{$\begin{array}{l}\text { Apoio emocional/ } \\
\text { informação }\end{array}$} & \multicolumn{2}{|c|}{ Apoio material } \\
\hline & $\mathrm{OR}^{*}$ & IC95\% & $\mathrm{OR}^{\star}$ & IC95\% & $O R^{*}$ & IC95\% \\
\hline \multicolumn{7}{|l|}{ Sexo } \\
\hline Feminino & 1,0 & - & 1,0 & - & 1,0 & - \\
\hline Masculino & 1,3 & $1,1-1,5$ & 0,9 & $0,8-1,0$ & 1,2 & $1,1-1,4$ \\
\hline \multicolumn{7}{|l|}{ Faixa etária (em anos) } \\
\hline$>40$ & 1,0 & - & 1,0 & - & 1,0 & - \\
\hline$\leq 40$ & 1,3 & $1,1-1,5$ & 1,2 & $1,1-1,4$ & 1,4 & $1,2-1,5$ \\
\hline \multicolumn{7}{|l|}{ Situação conjugal } \\
\hline Não casado & 1,0 & - & 1,0 & - & 1,0 & - \\
\hline Casado/companheiro & 2,1 & $1,8-2,4$ & 1,4 & $1,2-1,6$ & 1,3 & $1,1-1,5$ \\
\hline \multicolumn{7}{|l|}{ Escolaridade ${ }^{\star \star}$} \\
\hline Fundamental & 1,0 & - & 1,0 & - & 1,0 & - \\
\hline Médio & 1,4 & $1,2-1,7$ & 1,7 & $1,4-2,0$ & 1,7 & $1,5-2,1$ \\
\hline Superior & 1,8 & $1,5-2,1$ & 2,2 & $1,9-2,6$ & 2,6 & $2,2-3,0$ \\
\hline \multicolumn{7}{|l|}{ Renda per capita (em SM) } \\
\hline$<3$ & 1,0 & - & 1,0 & - & 1,0 & - \\
\hline $3-6$ & 1,2 & $1,0-1,4$ & 1,2 & $1,1-1,4$ & 1,3 & $1,1-1,5$ \\
\hline$>6$ & 1,8 & $1,5-2,1$ & 1,9 & $1,6-2,2$ & 1,9 & $1,6-2,2$ \\
\hline \multicolumn{7}{|l|}{ Número de parentes ou amigos íntimos ${ }^{\star \star}$} \\
\hline Nenhum & 1,0 & - & 1,0 & - & 1,0 & - \\
\hline $1-2$ & 3,3 & $2,2-4,9$ & 4,4 & $2,8-7,0$ & 2,9 & $2,0-4,3$ \\
\hline $3-4$ & 4,8 & $3,2-7,2$ & 7,9 & $5,0-12,5$ & 4,2 & $2,9-6,1$ \\
\hline $5-7$ & 7,0 & $4,7-10,4$ & 10,9 & $7,0-17,2$ & 5,2 & $3,6-7,5$ \\
\hline 8 ou mais & 10,3 & $6,9-15,4$ & 15,6 & $9,9-24,6$ & 7,1 & $4,9-10,1$ \\
\hline \multicolumn{7}{|l|}{ Mora só } \\
\hline Sim & 1,0 & - & 1,0 & - & 1,0 & - \\
\hline Não & 1,9 & $1,6-2,4$ & 1,5 & $1,2-1,8$ & 1,9 & $1,5-2,3$ \\
\hline \multicolumn{7}{|l|}{$\begin{array}{l}\text { Atividades esportivas e/ou artísticas } \\
\text { em grupo }{ }^{\star \star \star}\end{array}$} \\
\hline Não & 1,0 & - & 1,0 & - & 1,0 & - \\
\hline Sim & 1,6 & $1,4-1,8$ & 1,3 & $1,1-1,5$ & 1,3 & $1,1-1,5$ \\
\hline \multicolumn{7}{|l|}{ 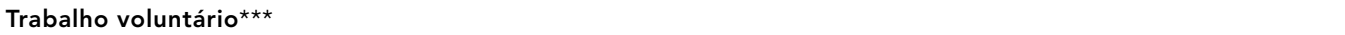 } \\
\hline Não & 1,0 & - & 1,0 & - & 1,0 & - \\
\hline Sim & 1,3 & $1,1-1,5$ & 1,3 & $1,1-1,6$ & 1,2 & $1,0-1,4$ \\
\hline \multicolumn{7}{|l|}{ Freqüência em atividades religiosas ${ }^{\star \star \star}$} \\
\hline 1 vez no ano ou nenhuma & $1,0 \#$ & - & $1,0 \#$ & - & 1,0 & - \\
\hline 2 a 3 vezes por mês ou algumas vezes no ano & 1,2 & $1,0-1,4$ & 1,2 & $1,0-1,4$ & 1,0 & $0,8-1,2$ \\
\hline 1 vez por semana ou mais & 1,2 & $1,0-1,5$ & 1,3 & $1,3-1,6$ & 1,0 & $0,9-1,2$ \\
\hline \multicolumn{7}{|l|}{ Número de doenças crônicas\# } \\
\hline 3 ou mais & 1,0 & - & 1,0 & - & 1,0 & - \\
\hline 1 ou 2 & 1,5 & $1,2-1,8$ & 1,4 & $1,2-1,7$ & 1,3 & $1,1-1,6$ \\
\hline Nenhuma & 1,8 & $1,5-2,2$ & 1,7 & $1,4-2,1$ & 1,7 & $1,4-2,1$ \\
\hline \multicolumn{7}{|l|}{ Estado de saúde auto-referido } \\
\hline Ruim/regular & 1,0 & - & 1,0 & - & 1,0 & - \\
\hline Bom/ótimo & 2,2 & $1,9-2,6$ & 2,2 & $1,8-2,6$ & 2,2 & $1,8-2,6$ \\
\hline \multicolumn{7}{|l|}{ Transtornos mentais comuns } \\
\hline Sim & 1,0 & - & 1,0 & - & 1,0 & - \\
\hline Não & 2,6 & $2,2-3,0$ & 2,2 & $1,9-2,5$ & 1,8 & $1,6-2,1$ \\
\hline
\end{tabular}

$\mathrm{SM}=$ salários mínimos

* OR = odds ratio;

** teste de tendência linear: $p<0,001$

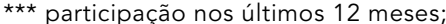

\# teste de tendência linear: $p<0,01$. 
de aferição de apoio social em nossa população de estudo 22 . Nos resultados apresentados aqui, a consistência interna da escala e de suas dimensões foi bastante elevada, e a retirada de qualquer dos itens não alterou significativamente esses valores. Todos os 19 itens apresentaram correlação excedendo o ponto de corte de 0,40 , recomendado como critério para exclusão do item da respectiva dimensão 28 . Além disso, para a grande maioria das perguntas, o coeficiente de correlação item-escala corrigido do item com o escore de sua dimensão foi pelo menos dois erros-padrão maior do que o mesmo coeficiente estimado entre aquele item e qualquer outra dimensão.

Em nossa população, apenas três dimensões foram discriminadas pela análise fatorial, em comparação às quatro identificadas por Sherbourne \& Stewart 18. Segundo esses autores, evidências empíricas da multidimensionalidade das escalas de apoio social têm sido controversas. Em alguns estudos, as dimensões são bastante correlacionadas e, portanto, de difícil distinção 31,32. Tal dependência é esperada, já que, em geral, as pessoas que recebem um determinado tipo de apoio também têm maior probabilidade de receberem outros tipos de ajuda. Logo, as perguntas representam dimensões de um mesmo constructo (apoio social) e são necessariamente correlacionadas. Em outros casos, entretanto, alguma evidência de independência entre as dimensões pôde ser demonstrada 18,29,30. A ênfase na necessidade de discriminar diferentes tipos de apoio está relacionada à possibilidade de que tenham importância distinta em relação a diversos desfechos de saúde 18.

Nossos resultados indicaram que os itens de apoio de interação social positiva não se diferenciaram dos itens de apoio afetivo, passando a constituir uma única dimensão (afetivo / interação social positiva). Isso sugere que pessoas que cumprem a função de apoio de interação social positiva representam mais do que uma companhia, ou alguém para fazer coisas agradáveis, sendo também alvos de sentimentos mais íntimos de afeto.

O fator que concentrou os itens da dimensão afetivo/interação social positiva incluiu dois itens originalmente alocados na dimensão emocional ("alguém para compartilhar preocupações e medos mais íntimos” e "alguém que compreenda seus problemas"). Esse achado parece indicar que a dimensão emocional não foi completamente capturada no contexto deste estudo, já que seus quatro itens foram divididos em aspectos afetivos/interação social positiva e emocional/informação. A decisão de mantê-los na dimensão da escala original emocional/informação foi baseada nas seguintes ponderações: (1) esses dois itens também apresentaram cargas altas no segundo fator, no qual se situaram as outras questões relativas ao apoio emocional e de informação; (2) um único teste empírico em uma população com características bem particulares (trabalhadores estáveis, sem grandes enfermidades) não pode ser suficiente para causar mudanças nas pertinências dos itens; (3) a correlação item-escala corrigida mostra correlações mais altas desses itens com a dimensão apoio emocional/informação.

Assim, como no estudo norte-americano 18, os itens relativos ao apoio emocional e de informação passaram a integrar uma única dimensão. É possível que a maneira como os itens foram construídos tenha influenciado na baixa capacidade de discriminação dessas duas dimensões, quando, por exemplo, procura captar apoio de informação através de "aconselhamento”, que, em geral, é adequado para necessidades muito pessoais 32 .

Ainda de maneira semelhante ao estudo de Sherbourne \& Stewart 18 , os itens de apoio material constituíram uma dimensão separada, indicando que a ajuda material não está necessariamente vinculada a aspectos afetivos e emocionais.

A grande maioria das hipóteses de associação entre características selecionadas e o apoio social, mencionadas na literatura, foram confirmadas em nosso estudo. Os funcionários com melhor percepção de seu próprio estado de saúde, os que referiram menor número de doenças crônicas e os classificados como negativos para transtornos mentais comuns tiveram chances mais elevadas de perceberem alto apoio social. Outras investigações também relataram maiores escores de apoio social entre indivíduos com melhor nível de saúde física e mental 9,18,29. É preciso notar a limitação do desenho transversal de nosso estudo para estabelecer a temporalidade dessas relações: tanto a percepção de pouca disponibilidade de apoio pode influenciar na pior avaliação do estado de saúde física e/ou mental, como diversos graus de doença ou mal-estar podem desencadear isolamento, e, portanto, menor nível de apoio social.

No presente estudo, as variáveis utilizadas para medir rede social estão associadas às dimensões de apoio, observando-se, nesse aspecto, relação forte, direta e consistente entre a quantidade de parentes e amigos íntimos referidos e níveis de apoio social em todas as dimensões. Nesse sentido, Bowling 16 sugere que 
o número de relacionamentos aumenta a probabilidade de apoio social, apesar de não garanti-lo. Associação menos evidente foi demonstrada entre participar de atividades esportivas e/ou artísticas em grupo ou participar de atividades de caráter voluntário e apoio social. Com relação à participação em atividades religiosas, encontramos associação com a dimensão de apoio emocional/informação e apoio afetivo/interação social positiva. Nesse aspecto, Helminen et al. 33 sugerem que pessoas que participam de atividades sociais em grupo tendem a ser mais apoiadas e menos vulneráveis ao isolamento, ao estresse e a problemas de saúde.

A associação entre viver sozinho e baixo apoio social, tal como encontramos em nossos resultados, também é relatada por outros autores $9,18,29,30,34$. Outro aspecto mencionado por esses autores e confirmado pelos nossos resultados diz respeito ao fato de que os participantes casados apresentam maior chance de perceber níveis mais altos de apoio, quando comparados aos não casados. Isso pode ser explicado pelo fato de que muitos indivíduos casados têm no seu cônjuge ou companheiro a principal fonte de apoio.

As outras hipóteses postuladas referem-se ao fato de que pessoas mais velhas têm percepção de menor apoio social, uma vez que o envelhecimento pode causar modificações e perdas de componentes da rede e, portanto, do apoio social disponível 35. De maneira semelhante ao estudo de Saameño et al. 29 , os funcionários mais velhos de nossa população apresentaram chance menor de referirem níveis altos de apoio social, quando comparados aos mais jovens. Além disso, percepções mais altas de apoio social foram identificadas entre os in- divíduos com níveis mais altos de escolaridade e renda per capita. Saameño et al. 29 encontraram resultados semelhantes e sugeriram que os indivíduos de nível sócio-econômico mais elevado sentem-se possivelmente mais independentes e auto-suficientes em sua vida social, percebendo-se, por isso, mais apoiados em situações de necessidade.

Em relação à associação entre gênero e apoio social, nossos resultados mostram padrão diferenciado para as três dimensões avaliadas: os homens tiveram chance mais elevada de referirem altos escores de apoio afetivo/interação social positiva e apoio material, e as mulheres, de apoio emocional/informação. Outros estudos não encontraram associação entre essas duas características 29,30.

O número elevado de hipóteses de associação confirmadas no estudo americano e em nossa investigação, a manutenção da multidimensionalidade (três fatores) da escala, a boa consistência interna e os resultados das análises prévias de confiabilidade teste-reteste permitem-nos afirmar que o instrumento de apoio social utilizado, após tradução e adaptação para o português, manteve propriedades psicométricas adequadas. Ademais, o número de perguntas desse instrumento, que permite sua aplicação rápida, a aparente facilidade de compreensão e a padronização do estilo de perguntas e respostas colaboram também para a eficiência na sua aplicação. Portanto, a utilização desse questionário em estudos futuros para a identificação dos efeitos do apoio social sobre condições de saúde, bem como sua interação com outros determinantes de interesse, é indicada nesta população e em populações com características semelhantes.

\section{Resumo}

Este artigo verifica a validade de constructo da escala de apoio social utilizada em um estudo de coorte de trabalhadores de uma universidade no Rio de Janeiro, Brasil (Estudo Pró-Saúde). Os dados foram obtidos na primeira etapa do estudo, realizada em 1999, na qual 4.030 funcionários técnico-administrativos completaram, no ambiente de trabalho, um questionário multidimensional e autopreenchível. Associações entre as dimensões de apoio social e características sócio-demográficas, relacionadas à saúde e ao bem-estar, foram avaliadas. Por meio da análise fatorial, foi possível discriminar três dimensões de apoio: interação social positivalapoio afetivo; apoio emocional/de infor- mação; e apoio material. Foram confirmadas as hipóteses de que indivíduos menos solitários, com melhor estado de saúde auto-referido, com relato de participação mais freqüente em atividades sociais em grupo e sem suspeição para transtornos mentais comuns teriam maior percepção de apoio social. Conclui-se que há evidencias de que o instrumento apresenta validade de constructo, indicando-se sua utilização em análises futuras, no âmbito do Estudo Pró-Saúde e em populações similares.

Apoio Social; Estudos de Coortes; Questionários 


\section{Colaboradores}

R. H. Griep realizou a revisão bibliográfica e análise e discussão dos resultados. Colaborou na adaptação transcultural da escala de apoio social e elaborou a versão preliminar do artigo. D. Chor colaborou no planejamento e implementação do Estudo Pró-Saúde, na revisão bibliográfica e na análise e discussão dos resultados, coordenou a adaptação transcultural da escala de apoio social. E. Faerstein coordenou o planejamento e implementação do Estudo Pró-Saúde, colaborou na análise e discussão dos resultados. C. S. Lopes coordenou o planejamento e implementação do Estudo Pró-Saúde e colaborou na discussão dos resultados. G. L. Werneck colaborou na análise e discussão dos resultados. Todos os autores participaram da elaboração da versão final do artigo.

\section{Agradecimentos}

A toda a equipe do Estudo Pró-Saúde, por sua contribuição nas etapas dos pré-testes, estudo-piloto e reteste do questionário.

\section{Referências}

1. Cassel J. An epidemiological perspective of psychosocial factors in disease etiology. Am J Public Health 1974; 64:1040-3.

2. Cobb S. Social support as a moderator of life stress. Psychosom Med 1976; 38:300-14.

3. Berkman LF, Syme SL. Social networks, host resistance, and mortality: a nine-year follow-up study of Alameda County residents. Am J Epidemiol 1979; 109:186-204.

4. House JS, Robbins C, Metzner HL. The association of social relationships and activities with mortality: prospective evidence for Tecumseh Community Health Study. Am J Epidemiol 1982; 116:123-40.

5. Hanson BS, Ostergren PO. Different social network and social support characteristics, nervous problems and insomnia: theoretical and methodological aspects on some results from population study "men born in 1914", Malmo, Sweden. Soc Sci Med 1987; 25:849-59.

6. Orth-Gomér K, Johnson JV. Social network interaction and mortality. A six year follow-up study of a random sample of the Swedish population. J Chronic Disease 1987; 40:949-57.

7. Kaplan G. Social connections and mortality from all causes and from cardiovascular disease: prospective evidence from Eastern Finland. Am J Epidemiol 1988; 128:370-80.

8. Vogt TM, Mullooly JP, Ernst D, Pope CR, Hollis JF. Social networks as predictors of ischemic heart disease, cancer, stroke and hypertension: incidence, survival and mortality. J Clin Epidemiol 1992; 45:659-66.

9. Oxman TE, Berkman LF, Kasl S, Freeman DH, Barrett J. Social support and depressive symptoms in the elderly. Am J Epidemiol 1992; 135:356-68.

10. Kawachi I, Colditz G, Ascherio A, Rimm EB, Giovannucci E, Stampfer MJ, et al. A prospective study of social networks in relation to total mortality and cardiovascular disease in men in the USA. J Epidemiol Community Health 1996; 50:245-51.

11. Avlund K, Damsgaard MT, Holstein BE. Social relations and mortality in eleven year follow-up study of 70-year-old men and women in Denmark. Soc Sci Med 1998; 47:635-43.

12. Fratiglione L, Wang HX, Ericsson K, Maytan M, Winblad B. Influence of social network on occurrence of dementia: a community-based longitudinal study. Lancet 2000; 355:1315-9.

13. O'Reilly P. Methodological issues in social support and social network research. Soc Sci Med 1988; 26:863-73.

14. Dean K, Holst E, Kreiner S, Schoenborn C, Wilson $R$. Measurement issues in research on social support and health. J Epidemiol Community Health 1994; 48:201-6.

15. McDowell I, Newell C. Measuring health: a guide to rating scales and questionnaires. 2nd Ed. New York: Oxford University Press; 1996.

16. Bowling A. Measuring social networks and social support. In: Bowling A, editor. Measuring health: a review of quality of life measurements scales. $2^{\text {nd }}$ Ed. Buckingham: Open University Press; 1997. p. 91-109. 
17. Due P, Holstein B, Lund R, Modvig J, Avlund K. Social relations: network, support and relational strain. Soc Sci Med 1999; 48:661-73.

18. Sherbourne CD, Stewart AL. The MOS social support survey. Soc Sci Med 1991; 38:705-14.

19. Payne RL, Jones G. Measurement and methodological issues in social support. In: Kasl C, Cooper CL, editors. Stress and health: issues in research methodology. New York: John Wiley \& Sons; 1987. p. 167-205.

20. Reichenheim ME, Moraes CL. Buscando a qualidade das informações em pesquisas epidemiológicas. In: Minayo MCS, Deslandes SF, organizadores. Caminhos do pensamento: textos de metodologia. Rio de Janeiro: Editora Fiocruz; 2002. p. 227-54.

21. Chor D, Griep RH, Lopes C, Faerstein E. Medidas de rede e apoio social no Estudo Pró-Saúde: prétestes e estudo-piloto. Cad Saúde Pública 2001; 17:887-96.

22. Griep Rh, Chor D, Faerstein E, Lopes C. Apoio social: confiabilidade teste-reteste de escala no Estudo Pró-Saúde. Cad Saúde Pública 2003; 19:62534.

23. Griep RH, Chor D, Faerstein E, Lopes C. Confiabilidade teste-reteste de aspectos da rede social no Estudo Pró-Saúde. Rev Saúde Pública 2003; 37: 379-85.

24. Griep RH. Confiabilidade e validade de instrumentos de medida de rede social e de apoio social utilizados no Estudo Pró-Saúde [Tese de Doutorado]. Rio de Janeiro: Escola Nacional de Saúde Pública, Fundação Oswaldo Cruz; 2003.

25. Streiner DL, Norman GR. Health measurement scales: a practical guide to their development and use. 2nd Ed. Oxford: Oxford University Press; 1995.

26. Faerstein E, Lopes CS, Valente K, Plá MAS, Ferreira MB. Pré-testes de um questionário multidimensional autopreenchível: a experiência do Estudo Pró-Saúde. Physis (Rio J) 1999; 9:117-30.

27. Norman GR, Streiner DL. Biostatistics: the bare essentials. 2nd Ed. London: B. C. Decker; 2000.
28. McHorney CA, Ware Jr. JE, Lu JFR, Sherbourne CD. The MOS 36-item short-form health survey (SF36). III. Tests of data quality, scaling assumptions, and reliability across diverse patient groups. Med Care 1995; 32:40-66.

29. Bellon-Saameño JAB, Sánchez AD, Castilho JDL, Claret PL. Validez y fiabilidad del cuestionario de apoyo social functional Duke - UNC - 11. Aten Primaria 1996; 18:153-63.

30. Broadhead WE, Gehlbach SH, Degruy FV, Kaplan BH. The Duke-UNC functional social support questionnaire: measurement of social support in family medicine patients. Med Care 1988; 26:70921.

31. House JS, Kahn R. Measures and concepts of social support. In: Cohen S, Syme S, editors. Social support and health. Orlando: Academic Press; 1985. p. 79-108.

32. Berkman LF, Glass T, Brissette I, Seeman TE. From social integration to health: Durkheim in the new millennium. Soc Sci Med 2000; 51:843-57.

33. Helminen A, Halonen P, Rankinen T, Nissinen A, Rauramaa R. Valitidy assessment of a social support index. Scand J Soc Med 1995; 23:66-74.

34. Umberson D. Gender, marital status and the social control of health behavior. Soc Sci Med 1992; 34:907-17.

35. Oslen O, Iversen L, Sabroe S. Age and the operationalization of social support. Soc Sci Med 1991; 32:767-71.

36. Goldberg DP, Williams PA. The user's guide to the General Health Questionnaire. Windsor: NferNelson; 1988.

37. Stanfeld SA, Marmot MG. Social class and minor psychiatric morbidity. A validated screening survey using the General Health Questionnaire in British civil servants. Psychol Med 1992; 22:739-49.

38. Mari JJ, Williams P. A comparison of the validity of two psychiatric screening questionnaires (GHQ12 and SRQ-20) in Brazil, using Relative Operating Characteristic (ROC) analysis. Psychol Med 1985; 15:651-9.

Recebido em 27/Nov/2003

Versão final reapresentada em 02/Set/2004

Aprovado em 21/Out/2004 